\title{
Editores
}

\section{Valmore Bermúdez-Pirela}

Yaneth Herazo-Beltrán

\section{Aspectos Básicos en Obesidad}

\section{Gimon pracuore N I V E R S I D A D digangis SIMON BOLIVAR




\title{
OBESIDAD Y ATEROSCLEROSIS
}

\author{
Fulio Acosta Martínez ${ }^{6}$
}

${ }^{6}$ Médico Cirujano por la Universidad Central de Venezuela. Médico Cardiólogo por la Universidad Central de Venezuela. Presidente de la Asociación Venezolana de Aterosclerosis y del Colegio Venezolano de Endotelio. Ex presidente de la Sociedad Venezolana de Cardiología. Orcid: bttps://orcid.org/0000-0002-4992-5202.e-mail: acostajulio@yahoo.com.

Palabras clave

Obesidad, aterosclerosis, inflamación, síndrome metabólico, obesidad abdominal

\section{Resumen}

El presente capítulo está referido a la relación entre obesidad y enfermedad aterosclerótica desde el punto de vista epidemiológico y etiopatogénico. otros capítulos describirán la asociación de obesidad con los factores de riesgo. en registros de mortalidad de pacientes obesos, la primera causa de muerte es de origen vascular, superior a neoplasias y afecciones respiratorias o renales. El índice de masa corporal (IMC) y la relación cintura/ cadera se correlacionan con prevalencia e incidencia de eventos cardiovasculares; el IMC es marcador de progresión de placas. La inflamación es un componente importante de la enfermedad cardiovascular aterosclerótica, involucrada en todas las etapas del proceso, desde el inicio hasta sus complicaciones; los pacientes obesos tienen mayor concentración de los marcadores de inflamación vascular sistémica y de éstas con la incidencia de eventos cardíacos y cerebrales. Aumento de la grasa corporal total y abdominal se acompaña de estrés oxidativo y disfunción endotelial. Puede esperarse que la reducción terapéutica de la obesidad se asocie a disminución de los mecanismos aterogénicos y de la incidencia de eventos clínicos cardiovasculares de origen aterosclerótico.

\section{OBESITY AND ATHEROSCLEROSIS}

\section{Keywords}

Obesity, Atherosclerosis, Inflammation, metabolic syndrome, abdominal obesity

\section{Abstract}

This chapter refers to the relationship between obesity and atherosclerotic disease from the epidemiological and etiopathogenic point of view. Other chapters will describe the association of obesity with Risk Factors. In registries of mortality of obese patients, the first 
cause of death is of vascular origin, superior to neoplasms and respiratory or renal affections. The Body Mass Index (BMI) and the Waist / Hip Ratio correlate with the prevalence and incidence of Cardiovascular Events; the BMI is a marker of plate progression. Inflammation is an important component of atherosclerotic cardiovascular disease, involved in all stages of the process, from the beginning to its complications; obese patients have a higher concentration of markers of systemic vascular inflammation and of these with the incidence of cardiac and cerebral events. Increased total and abdominal body fat is accompanied by oxidative stress and endothelial dysfunction. It can be expected that the therapeutic reduction of obesity is associated with a decrease in atherogenic mechanisms and the incidence of cardiovascular clinical events of atherosclerotic origin.

\section{INTRODUCCIÓN}

Los síndromes clínicos potencialmente fatales asociados a la enfermedad aterosclerótica constituyen en muchos países la primera causa de muerte. En Venezuela las enfermedades cardiovasculares ocupan el primer lugar como causa de muerte en la población adulta, de ellas las formas clínicas de presentación de la isquemia miocárdica y cerebral de origen aterosclerótico son las predominantes (Flegal, 2005).

Los parámetros biológicos usados para analizar la relación entre la obesidad y la enfermedad cardiovascular de origen aterosclerótico son variables y se utilizan aislados o combinados en los estudios publicados; de igual manera difieren los puntos de corte para la definición de la obesidad.

\section{Tabla 1. Índices de obesidad}

\begin{tabular}{l}
\hline Índices de obesidad \\
\hline Peso corporal \\
Índice de masa corporal \\
Circunferencia abdominal \\
Relación circunferencia abdominal/circunferencia de la cadera \\
Relación circunferencia abdominal/altura \\
Indice de grasa corporal \\
Composición tisular corporal \\
\hline
\end{tabular}


Una importante cantidad de estudios epidemiológicos ha descrito la relación entre la obesidad y la enfermedad cardiovascular, específicamente en hipertensión arterial, insuficiencia cardíaca, arritmias cardíacas, enfermedad coronaria y vascular cerebral; más numerosos aún son aquellos que estudian su relación con los denominados factores de riesgo para enfermedad cardiovascular o con enfermedad coronaria; el presente capítulo se refiere a la obesidad en general y la enfermedad aterosclerótica. Los resultados del Prospective Studies Collaboration mostraron la relación entre el Índice de Masa Corporal (IMC) y mortalidad total y por causas específicas obtenida de 57 estudios prospectivos con 894.576 participantes de Europa Occidental y Norteamérica, 61\% hombres, todos con edad promedio de 46 años $( \pm 11)$ reclutados de 1975 a 1985, que tenían IMC promedio de 25 (DE 4) kg/m2 en análisis ajustado para edad, género y consumo de tabaco que excluyó los primeros 5 años y se utilizó la evolución de los siguientes 8 años; la edad promedio a la muerte fue de 67 años +-10 (Prospective Studies Collaboration, 2009).

\section{Tabla 2. Causas de muerte en los pacientes obesos}

\begin{tabular}{cl}
\hline Causas de muerte en los pacientes obesos & \\
\hline Vascular & 30.416 \\
Neoplasia & 22.592 \\
Respiratoria & 3.770 \\
Diabetes, renal o hepática & 2.070 \\
Otras & 7.704 \\
\hline
\end{tabular}

En ambos sexos la menor mortalidad correspondió al IMC de 22,5 a $25 \mathrm{~kg} / \mathrm{m} 2$, luego por cada incremento de $5 \mathrm{~kg} / \mathrm{m} 2$ se registró un $30 \%$ de aumento en la mortalidad total y $40 \%$ de la mortalidad cardiovascular. Tabla 2.

\section{Obesidad y riesgo de infarto de miocardio}

En el estudio INTERHEART: Obesity an the risk of myocardial infarction in 27.000 participans from 52 countries: a case-contorl study, con 12.461 casos de 
Infarto de miocardio (IM) fueron comparados con 14.637 controles mediante análisis del IMC, circunferencia abdominal (CA) circunferencia de la cadera (CC) y la relación CA/CC (Yusuf, 2005), tabla 3.

\section{Tabla 3.}

\section{Relación cintura/cadera y riesgo relativo de evento $\mathrm{CV}$}

\begin{tabular}{ccc}
\hline Quintil & OR & IC 95\% \\
\hline $5^{\circ}$ & 2,52 & $2,31-2,74^{*}$ \\
$4^{\text {o }}$ & 1,90 & $1,74-2,07$ \\
$3^{\text {o }}$ & 1,39 & $1,28-1,52$ \\
$2^{\text {o }}$ & 1,15 & $1,05-1,26$ \\
$1^{\text {o }}$ & Grupo de & \\
& referencia &
\end{tabular}

${ }^{*} \mathrm{p}<0,0001$ quintil $5^{\circ}$ vs quintil $1^{\circ}$

ajustado para edad, sexo, tabaco y región.

\section{Relación índice de masa corporal y eventos cardiovasculares}

En el mismo estudio INTERHEART fue descrita la correlación del IMC con el IM y el riesgo (OR) no ajustado se encontró en 1,44 (IC95\% 1,32-1,57) en el quintil mayor vs el menor. Al ajustarlo con la relación CA/ CC la relación fue menor (OR 1,12 IC 1,03-1,22) e insignificante al ajustarlo con los otros factores de riesgo (OR 0,98 IC 0,88-1,09).

En otro estudio realizado en población francesa, Audureau publicó los resultados del Estudio Nacional de Calidad de Vida realizado en 10.305 encuestados, publicado en el 2016. La relación entre el índice de masa corporal y la prevalencia de enfermedad cardiovascular (Cardiopatía isquémica y enfermedad vascular cerebral y periférica) se encontró distinta para diferentes edades, pero siempre mayor en comparación con los grupos no obesos y el mayor impacto se observó en los mayores de 35 años, Tabla 4, (Audureau, 2016). 


\section{Tabla 4.}

Relación entre el IMC y la prevalencia de enfermedad cardiovascular

\begin{tabular}{lcccccc}
\hline IMC Kg/m2 & \multicolumn{7}{c}{ Grupos etarios } \\
\hline IMC & $<18,5$ & $18,5 \mathrm{a}<25$ & $25 \mathrm{a}<30$ & $30 \mathrm{a}<35$ & $>35$ & $\mathrm{p}$ \\
\% Evento CV & 2,1 & 1,5 & 2,1 & 2,9 & 5,9 & $<0,0001$ \\
\hline
\end{tabular}

\section{Obesidad y progresión de aterosclerosis.}

En otro estudio de los pacientes del Randomized Trial of Imagen vs Risk Factors-based Therapy for Plaque Regression $\div$ se investigaron los cambios del volumen de placas ateroscleróticas carotídeas mediante imágenes basales y a 12 meses obtenidas por resonancia magnética (RMI) en 106 pacientes tratados con estatina; $62 \%$ hombre, edad promedio 65 años, clasificados según el IMC. Todos fueron tratados con estatina y tuvieron una reducción similar de las concentraciones de LDL-C, la presión arterial se mantuvo controlada en valores normales; el riesgo de enfermedad coronaria estimado según el puntaje de Framingham era 8\% en 10 años. En esta población de pacientes de riesgo moderado tratados con estatina, la obesidad se encontró asociada al progreso del volumen de las placas ateroscleróticas en comparación con los pacientes no obesos. La probabilidad de crecimiento de las placas fue mayor en los pacientes obesos. Tablas 5 y 6 .

\begin{tabular}{llll}
\multicolumn{4}{c}{ Tabla 5.} \\
Cambio del volumen de la placa & y obesidad \\
\hline Peso & IMC kg/m2 & $\%$ de cambio de la placa \\
\hline Normal & $18,5 \quad \mathrm{a}<25$ & $-4,2$ & $\mathrm{p}<0,05$ \\
Sobrepeso & $25 \mathrm{a}<30$ & $-3,5$ & \\
Obeso & $30+$ & $+4,8$ & \\
\hline
\end{tabular}

Tabla 6. Progresión de la placa aterosclerótica y obesidad

\begin{tabular}{cc}
\hline \% de pacientes & \\
\hline Obesos & 70 \\
No obesos & 34 \\
\hline
\end{tabular}




\section{Inflamación}

Las evidencias indican que la aterosclerosis es una enfermedad inflamatoria y que la inflamación es un componente importante de la enfermedad cardiovascular aterosclerótica. En todas las etapas del proceso aterosclerótico está involucrada la inflamación subclínica, desde el inicio de la infiltración de la pared y la formación de la estría grasa hasta la ruptura y trombosis de una placa o la obstrucción completa, ambas situaciones que conducen al infarto del miocardio o la muerte súbita. En el estudio sobre la relación entre la obesidad y el progreso de la placa aterosclerótica también se analizó la concentración sérica de proteína $\mathrm{C}$ reactiva evaluada con método de alta sensibilidad en pacientes obesos y no obesos que estaban en tratamiento con estatinas, y se encontró mayor concentración del marcador de inflamación subclínica en los pacientes obesos. Tabla 7.

\section{Tabla 7. \\ Concentración de proteína $\mathrm{C}$ reactiva promedio en pacientes obesos y no obesos}

\begin{tabular}{|l|l|l|}
\hline Obesos & $1,84 \mathrm{mg} / \mathrm{L}$ & $\mathrm{p}<0,0001$ \\
\hline No obesos & 0,77 & \\
\hline
\end{tabular}

En un estudio publicado más recientemente, realizado en 6.075 pacientes masculinos de 28 a 61 años $(46,8+-3,7)$ de Malmö, Suecia, seguidos por 18,7 años $(+-4,2)$ desde el registro de pacientes sanos hasta un evento cardiovascular, con el propósito de investigar la relación entre la obesidad, los marcadores de inflamación subclínica y el riesgo de eventos cardiovasculares. La obesidad fue determinada mediante el índice de masa corporal (IMC) de $30 \mathrm{~kg} / \mathrm{m} 2$ o mayor; ausencia de obesidad en los pacientes con IMC de 25 o menos; la inflamación fue establecida mediante la determinación de las concentraciones de proteínas plasmáticas sensibles a inflamación (PSI) y los eventos cardiovasculares fueron: a) Cardíacos: infarto del miocardio o muerte por isquemia miocárdica y b) Cerebrales: ictus fatal y no fatal por hemorragia subaracnoidea, intracerebral o por isquemia. También fueron divididos en alto o bajo nivel de factores de riesgo tradicionales: los de bajo nivel no tenían 
hipertensión, diabetes, dislipidemia o tabaco. Los autores encontraron que las concentraciones de las PSI: Fibrinógeno, Haptoglobina y Orosomucoide (alfa1-glicoproteina ácida) mostraron correlación positiva y lineal con el IMC. El 41,6 \% de los obesos tuvieron elevación de las tres PSI y en los no obesos el 33,6\%. Tabla 8

\section{Tabla 8.}

Riesgo relativo de eventos cardiovasculares ajustado para la edad y FR en pacientes obesos según el número de PSI con valores anormales altos; $\mathrm{p}$ 0,002 para las tendencias.

\begin{tabular}{|c|c|c|c|c|}
\hline PSI & \multicolumn{2}{|c|}{$\begin{array}{c}\text { Evento } \\
\text { cardiovascular }\end{array}$} & $\begin{array}{l}\text { Evento } \\
\text { cardíaco }\end{array}$ & $\begin{array}{c}\text { Evento } \\
\text { cerebral }\end{array}$ \\
\hline Número & \multicolumn{2}{|c|}{ RR (IC 95\%) } & $\%$ & $\%$ \\
\hline 0 & 2,1 & $(1,4-3,4)$ & 10,9 & 4,4 \\
\hline 1 & 2,4 & $(1,5-3,7)$ & 12,7 & 5,9 \\
\hline 2 & 3,7 & $(2,3-6,0)$ & 19,2 & 11,0 \\
\hline 3 & 4,5 & $(3,0-6,6)$ & 23,9 & 5,5 \\
\hline $\mathrm{p}$ & 0,00 & & 0,002 & ns \\
\hline
\end{tabular}

Los pacientes obesos no inflamados, sin elevación de los valores plasmáticos de las PSI, tuvieron mayor incidencia de eventos cardiovasculares y de eventos cardíacos en comparación con los pacientes no obesos sin inflamación, lo cual sugiere que la obesidad determinada por el IMC aumenta el riesgo de eventos cardíacos: infarto del miocardio y muerte cardíaca isquémica, aun en ausencia de marcadores de inflamación sistémica subclínica, riesgo que está aumentado de manera proporcional cuando hay inflamación, independiente de la presencia de otros factores de riesgo tradicionales (Enström, 2004).

\section{Estrés oxidativo}

La obesidad puede alterar la expresión por la célula endotelial vascular, de proteínas que aumentan la susceptibilidad de aterosclerosis. El endotelio 
ejerce función normal protectora anti-aterosclerótica, entre otras mediante la biodisponibilidad de Óxido Nítrico (ON), la inhibición de la producción de Especies Reactivas de Oxígeno (EROs) y del estrés oxidativo, una acción anti-inflamatoria y la reducción en la producción local de moléculas vasoconstrictoras. La producción experimental de obesidad se asocia al desarrollo de aterosclerosis; el aumento de la grasa corporal total y abdominal está ligada a alteración de la vasodilatación dependiente del endotelio, del estrés oxidativo y la producción aumentada de proteínas vasoconstrictoras como la Endotelina-1 (E-1). Silver y colaboradores investigaron en humanos la relación entre adiposidad y la expresión endotelial vascular de proteínas involucradas en la biodisponibilidad del ON (Silver, 2007).

\section{Tabla 9.}

\section{Proteínas involucradas en la biodisponibilidad de $\mathrm{ON}$}

Sintasa Endotelial del ON (eNOS).

eNOS fosforilada (P-eNOS).

Sistema de enzimas oxidantes: NAD(P)H oxidasa y la Xantina oxidasa.

Modificación oxidativa de proteinas (Nitrotyrosina).

Enzimas antioxidantes: Catalasa, la Superóxido-dismutasa citosólica (CuZnSOD) y la mitocondrial.

Molécula pro-inflamatoria Factor Nuclear Kapa-Beta (NF-kB), la Ciclo-oxigenasa-2.

Proteína vasoconstrictora E-1.

Para la investigación fueron cultivadas células endoteliales de 108 pacientes adultos normotensos, edad media 40 años +-2, 60\% masculinos, sin enfermedad cardiovascular ni diabetes u otra condición patológica. Los pacientes tenían varias magnitudes del Índice de Masa Corporal (IMC), de la Masa Grasa Total (GMT) y de circunferencia abdominal (CA); las proteínas fueron determinadas por inmunofluorescencia cuantitativa. Todas las medidas de adiposidad se encontraron asociadas positivamente con la producción de $\mathrm{NAD}(\mathrm{P}) \mathrm{H}$ oxidasa y catalasa. Estas dos moléculas más nitrotirosina y P-eNOS fueron mayores (entre 35 a 130\%) en los pacientes con sobrepeso/obesidad; también fue mayor la expresión de endotelina-1. Esta relación fue reducida alrededor de un $40 \%$ cuando se ajustó a la concentración de triglicéridos, 
proteína C reactiva, insulina en ayunas y el modelo de resistencia a la insulina, pero no sufrió modificación con el ajuste para otros lípidos plasmáticos, presión arterial o la glicemia en ayunas.

\section{Tabla 10.}

\section{Coeficiente de correlación de la producción de la $\mathrm{NAD}(\mathrm{P}) \mathrm{H}$ oxidasa-p47phox con los índices de obesidad}

\begin{tabular}{l|l}
\hline Índice de Masa Corporal & 0,24 \\
\hline Grasa Corporal Total & 0,23 \\
\hline Circunferencia Abdominal & 0,23 \\
\hline
\end{tabular}

La producción de endotelina 1 por el endotelio de los pacientes obesos fue $54 \%$ mayor que en el endotelio de las personas sin obesidad. La producción de NF kappa beta fue 60 a 100\% mayor en el endotelio cultivado de los pacientes del más alto quintil del IMC comparado con el más bajo de IMC y de circunferencia abdominal. La expresión de proteínas aterogénicas se asoció mejor con el contenido de la grasa abdominal medida por la absorción de rayos $\mathrm{x}$, pero no fue evidente la diferenciación entre IMC y circunferencia abdominal. En resumen, hay demostración de una relación entre la obesidad y la alteración de la expresión molecular en células endoteliales en el sentido de mayor producción de proteínas que favorecen al proceso aterogénico a través de aumento del estrés oxidativo y mediadores de la inflamación y alteración del estado de vasodilatación normal.

\section{Alteración de la microcirculación}

La disfunción endotelial está presente en el origen del proceso aterosclerótico, a lo largo de su progreso y en las complicaciones clínicas. En 108 pacientes se analizó la función vasodilatadora dependiente del endotelio mediante la prueba de hiperemia reactiva inducida por roce y evaluada con tres procedimientos: tonometría vascular digital, medida del flujo sanguíneo digital por el método doppler y la monitorización de la temperatura cutánea digital. Los tres procedimientos evalúan el efecto de la regulación endotelial sobre la microcirculación. En los pacientes con índice de masa corporal mayor de $30 \mathrm{~kg} / \mathrm{m} 2$ los resultados de los tres procedimientos fueron consistentes con 
una reducción de la respuesta vasodilatadora en la microcirculación inducida con el estímulo mecánico del roce propio de la prueba. Esta asociación de la obesidad con la disfunción de la microcirculación no estuvo presente cuando se correlacionó el resultado de la prueba con los factores de riesgo clásicos: hipertensión arterial, diabetes, hipercolesterolemia y tabaco, condiciones que si están asociadas con la disfunción de la microcirculación dependiente del endotelio. En los pacientes con diagnóstico de enfermedad coronaria la alteración de la microcirculación se encontró más pronunciada (Van del Heijden, 2017).

En resumen, las evidencias clínicas y epidemiológicas muestran consistentemente una relación positiva entre los parámetros clínicos utilizados para el diagnóstico de la obesidad con los eventos clínicos asociados a la enfermedad vascular aterosclerótica. Estas evidencias han sido respaldadas por los estudios de investigación animales y humanos en los cuales la obesidad está asociada con los mecanismos biológicos, bioquímicos y celulares relacionados patogénicamente con la enfermedad aterosclerótica y sus complicaciones, cambios que pueden modificarse favorablemente con el tratamiento de la obesidad y de esa manera poder eventualmente reducir los eventos clínicos; este aspecto necesita una mayor cantidad de estudios confirmatorios.

\section{Cómo citar este capítulo}

\section{APA}

Acosta-Martínez,J.(2018). Obesidady aterosclerosis. En Bermúdez-Pirela,V.,yHerazo-Beltrán, Y(Ed.), Aspectos básicos en obesidad (pp.60-70). Barranquilla, Colombia: Ediciones Universidad Simón Bolívar.

\section{VANCOUVER}

Acosta-Martínez J. Obesidad y aterosclerosis. En: Bermúdez- Pirela, V, Herazo-Beltrán, Y. (Editores.), Aspectos básicos en obesidad. Barranquilla:Ediciones Universidad Simón Bolívar; 2018. Pp.60-70.

\section{REFERENCIAS}

Audureau E, Pouchot J, Coste J. (2016) Gender-Related Differential Effects of Obesity on Health- Related Quality of Life via Obesity-Related 
Comorbidities A Mediation Analysis of a French Nationwide Survey. Circ Cardiovasc Qual Outcomes. 9:246-256.

Enström G, Heblad B, Stavenow L y col. (2004). Incidence of obesityassociated cardiovascular disease is related to inflammation-sensitive plasma proteins: a population-based cohort study. Arterioscler Throm Vasc Biol. 24:1498-1502.

Flegal KM, Gaubard BI, Williamson DF, Gail MH. (2005) Excess deaths associated with underweight, overweight and obesity. JAMA. 293:18611867.

Prospective Studies Collaboration (2009). Body-mass index cause-especific mortality in 900000 adults: collaborative analyses of 57 prospective studies. The Lancet. 373:1083-1096.

Silver A, Beske S, Christou D y col. (2007) Overweight and Obese Humans Demonstrate Increased VASCULAR endotelial NAD(P)H Oxidasep47phox Expression and Evidence of Endothelial Oxidative Stress. Circulation 2007;115:627-637.

Van del Heijden D, Van Leuven M, Janssens g y col. (2017). Body Mass Index is Associated with Microvascular Endothelial Dysfunctin in Patients with Treated Metabolic Risk Factors and Suspected Coronary Artey Disease. JAHA 2017:6;1-21.

Yusuf S, Hawken S, Ounpuu S, y col (2005). Obesity and the risk of myocardial infarction in 27000 oarticipants from 52 countries: a case-control study. The Lancet. 366:1640-1649. 
La obesidad es una enfermedad crónica caracterizada por un aumento de la grasa corporal expresada como una expansión del volumen del tejido adiposo por encima de los niveles considerados normales para la edad, sexo y etnicidad.Con frecuencia, la acumulación de grasa en las personas con obesidad ocurre de forma ectópica en órganos como el músculo y el hígado, lugares donde es capaz de producir alteraciones metabólicas como la resistencia a la insulina.

La Organización Mundial de la Salud, ha catalogado a la obesidad como un problema de salud pública en el ámbito mundial, que ha alcanzado proporciones epidémicas, reconociéndola como la enfermedad metabólica más frecuente en el ser humano. De hecho, su prevalencia se ha triplicado desde 1975 al 2016, de forma que para este último año había más de 1900 millones de personas con sobrepeso y más de 650 millones eran portadores de esta enfermedad.

Aunque anteriormente se consideraba un problema de países de altos ingresos, en la actualidad, la obesidad también es muy frecuente en muchos países de ingresos bajos y medianos. Esto ha llevado a que la OMS, diseñara la estrategia mundial para la prevención y control de las enfermedades no transmisibles, que constituye un mapa para el establecimiento de iniciativas de vigilancia, prevención y tratamiento de enfermedades como la obesidad, pero que a pesar de su claridad, no ha recibido la atención suficiente por parte de los gobiernos y la colectividad en general con el propósito de logran un alto impacto en la prevención de la obesidad. 